\title{
Relación entre los Ensayos Ultrasónico y Convencional para determinar la Resistencia del Concreto Simple a la Compresión*
}

\section{Correlation between Ultrasonic and Conventional Tests in Order to Determinate the Compression Strength of Plain Concrete}

DOI: http://dx.doi.org/10.17981/ingecuc.12.1.2016.10

Artículo de Investigación Científica - Fecha de Recepción: 09 de Septiembre de 2015 - Fecha de Aceptación: 07 de Diciembre de 2015

\section{Jorge Enrique Cruz Benedetti}

Especialista en Análisis y diseño de estructuras y en Gerencia de Proyectos de Construcción. Universidad Pontificia Bolivariana. Montería (Colombia). jecruzb@gmail.com

\section{Luisa Martínez Acosta}

Magister en Ingeniería Civil. Universidad Pontificia Bolivariana. Montería (Colombia). ingluisamartinez@gmail.com

\section{Álvaro López Ramos}

Magister en Hidrología. Universidad Pontificia Bolivariana. Montería (Colombia). alvaro.lopezr@upb.edu.co

Jaime Andrés Lakah Durango

Especialista en Gerencia para Ingenieros. Universidad Pontificia Bolivariana. Montería (Colombia). jaime.lakah@upb.edu.co

Claudia Ceballos Díaz

Magister en Ingeniería Civil. Universidad Pontificia Bolivariana. Montería (Colombia). claudia.ceballos@upb.edu.co

Para citar este artículo / To reference this paper:

J. E. Cruz Benedetti, L. Martínez Acosta, Á. López Ramos, J. A. Lakah Durango y C. Ceballos Díaz "Relación entre los Ensayos Ultrasónico y Convencional para Determinar la Resistencia del Concreto Simple a la Compresión", INGE CUC, vol. 12, no. 1, pp. 94-98, 2016. DOI: http://dx.doi.org/10.17981/ingecuc.12.1.2016.10

\begin{abstract}
Resumen-- Los ensayos de resistencia de especímenes de concreto por el método de ondas ultrasónicas contienen muchas variables tales como tamaño de los agregados, relación agua cemento, temperatura, densidad de los materiales, entre otros, que inciden en la resistencia final del espécimen. Sin embargo, se ha constituido en un método no destructivo rápido para diagnosticar resistencias de estructuras ya construidas y detectar anomalías tales como fisuras o discontinuidades. En este artículo se halla una correlación entre las lecturas de las ondas ultrasónicas y la resistencia a la compresión de concretos de 17.5, 21, 24.5 y $28 \mathrm{MPa}$ para 7 y 28 días, con diferentes agregados gruesos y finos de la ciudad de Montería, de manera que se pueda diagnosticar la resistencia del concreto de las estructuras antiguas que no cumplan con la Reglamento Colombiano de Construcción Sismo Resistente - NSR 10.
\end{abstract}

Palabras claves-- agregados pétreos; resistencia a la compresión; ultrasonido.

\begin{abstract}
Strength tests of concrete specimens by the method of ultrasonic waves contain many variables such as the size of aggregate, water cement ratio, temperature, density of materials, among others, that affect the final strength of the specimen. However, it has become a fast nondestructive method to diagnose resistant of already built structures and detect anomalies such as cracks or discontinuities. This article finds a correlation between the readings of the ultrasonic waves and the compressive strength of concrete at 17.5, 21, 24.5 and 28 $\mathrm{MPa}$ for 7 and 28 days, with varying coarse and fine aggregates of Montería city so as to diagnose the concrete strength of the ancient structures that do not comply with the Regulation Colombian Earthquake Resistant Construction - NSR 10.
\end{abstract}

Keywords-- Petrous Aggregates; Strength Compressive Test; Ultrasound.

*Artículo de investigación científica derivado del proyecto de investigación titulado: "Estudio de correlación entre las lecturas de velocidad de onda de un instrumento de ultrasonido y la resistencia a la compresión de especímenes de concreto con diferentes dosificaciones de materiales de la región”. Financiado por la Universidad Pontificia Bolivariana seccional Montería. Fecha de inicio: julio de 2010. Fecha de finalización: diciembre de 2011. 


\section{INTRODUCCIÓN}

Existen múltiples métodos para la determinación de la resistencia a la compresión del concreto simple, los cuales se clasifican en destructivos y no destructivos y que dependen de la conservación de la integridad del espécimen empleado en la realización del ensayo; entre los primeros el más utilizado es el de rotura de cilindros con la máquina de compresión. La resistencia a la compresión se obtiene dividiendo la fuerza aplicada entre el área de la sección transversal de la probeta.

Existen diversos métodos no destructivos para determinar la resistencia del concreto, entre los cuales se pueden enumerar: uso del ultrasonido para la reconstrucción de una imagen en 3D del espécimen a estudiar [1]; correlación de VPU (Velocidad de Pulso Ultrasónico) con redes neuronales, las cuales dan unas predicciones confiables de la resistencia [2]; ultrasonido con Amplitud del Pulso Ultrasónico (APU) [3], entre otros. Para este estudio se escogió la utilización del método no destructivo del ultrasonido VPU, el cual es más utilizado que el APU, mediante el cual se obtienen lecturas del tiempo que demora una onda al recorrer una distancia a través del concreto, y que guardan relación con la resistencia del mismo.

Conociendo que la resistencia del concreto depende entre otros factores del tipo de agregados pétreos utilizados en su elaboración [4,5], se plantea hallar una correlación estadística apropiada entre el pulso ultrasónico y el ensayo a compresión del concreto simple con diferentes tipos de agregados de la región de Córdoba para diferentes resistencias, específicamente de la ciudad capital Montería, la cual presenta una actividad constructora en auge y que también cuenta con construcciones vetustas declaradas patrimonio arquitectónico y que por lo tanto deberán potencializarse para cumplir con el reglamento colombiano de construcción sismo resistente (NSR - 10) [6]. La ciudad está asentada a orillas del Río Sinú, el cual proporciona unos excelentes agregados pétreos (canto rodado y arena de alto módulo de finura), para la actividad constructora que tradicionalmente se han utilizado con una dosificación en volumen de 1:2:3 consiguiéndose fácilmente unas resistencias superiores a los $28 \mathrm{MPa}$. En los últimos años, y debido a la demanda de las obras civiles se está utilizando piedra caliza triturada de canteras aledañas al $\mathrm{Mu}$ nicipio de Montería que forma parte del sistema de serranías terminales de la Cordillera Occidental de Los Andes Colombianos y que está clasificado como depósitos aluviales y llanuras aluviales pertenecientes a la era Cenozoica, periodo cuaternario (Qal) [7]

Además de obtener la resistencia a la compresión de elementos de concreto reforzado (CR), se podrán detectar fisuras, discontinuidades y delaminaciones por la diferencia de lecturas en zonas cercanas una de la otra [7].

Se ha comprobado en estudios previos que las grietas de los especímenes sometidos al ensayo de com- presión estándar aparecen cuando se le ha aplicado el $85 \%$ de la carga de rotura y la velocidad del pulso ha bajado a un $94 \%$ [13].

Existen otros estudios similares al propuesto en este artículo, en donde solamente se ha relacionado concretos elaborados con agregados de caliza mediante la utilización del pulso ultrasónico [17].

En este estudio se amplió el rango de materiales pétreos de acuerdo al número de combinaciones de resumidos en el cuadro 1, de materiales obtenidos en la zona de estudio:

Tabla I. Combinaciones de agregados para las mezclas

\begin{tabular}{|c|c|c|c|c|c|}
\hline Grupo & $\begin{array}{c}\text { Arena } \\
\text { fina }\end{array}$ & $\begin{array}{c}\text { Arena } \\
\text { gruesa }\end{array}$ & Caliza & $\begin{array}{c}\text { Canto } \\
\text { rodado }\end{array}$ & $\begin{array}{c}\text { Caliza+ } \\
\text { canto } \\
\text { rodado }\end{array}$ \\
\hline 1 & $\mathrm{x}$ & & $\mathrm{x}$ & & \\
\hline 2 & $\mathrm{x}$ & & & $\mathrm{x}$ & \\
\hline 3 & $\mathrm{x}$ & & & & $\mathrm{x}$ \\
\hline 4 & & $\mathrm{x}$ & $\mathrm{x}$ & & \\
\hline 5 & & $\mathrm{x}$ & & $\mathrm{x}$ & \\
\hline 6 & & $\mathrm{x}$ & & & $\mathrm{x}$ \\
\hline
\end{tabular}

Fuente: Autor

\section{Metodología}

a) Caracterización de los agregados pétreos y diseño de mezclas: se hicieron análisis granulométricos de suelos por tamizado (INV. E-123-07) [8] y masa unitaria suelta y compacta de los agregados (NTC 237 y NTC 176) [9], [10]. Se hizo un diseño de mezcla de conformidad con lo establecido en ACI-211, para dosificar concreto normal y pesado.

El diseño de mezcla fue hecho para resistencias de $17.5,21.0,24.5$ y $28.0 \mathrm{MPa}$, usando como agregados pétreos arena fina, arena gruesa, piedra caliza de cantera y grava o canto rodado del Río Sinú y una relación agua cemento alrededor de 0.45 en promedio [11]. Para la mezcla de caliza y Canto rodado de las combinaciones 3 y 6 , se utilizó una proporción de $50 \%$ para cada una [12].

b) Pruebas de ultrasonido y rotura de probetas: Los especímenes utilizados para este estudio tenían un diámetro de $150 \mathrm{~mm}$ y una altura de $300 \mathrm{~mm}$, medidas estándar para un cilindro de prueba de concreto con una relación alto/ancho de 2 .

Se tomaron datos de velocidad de pulso por transmisión directa que arroja resultados más precisos; se rompieron las probetas en la máquina de compresión estándar y se obtuvieron datos de resistencia a la compresión a los 7 y 28 días de edad, para las cuatro resistencias propuestas.

Los datos obtenidos se tabularon y graficaron con respecto a su edad en dos esquemas de velocidad vs resistencia y a través del análisis estadístico de estos datos se establecieron los valores de correlación apropiados [14]. 
El número de probetas diseñadas y falladas para cada combinación fue de ochenta (80), divididos en grupos de cuarenta (40) para dos edades de 7 y 28 días respectivamente con resistencias de diseño 17.5, 21.5, 24 y 28.5 Mpa; se limitó este proyecto de investigación al rango anteriormente descrito porque se conoce por estudios previos que para concretos de alto desempeño las pruebas ultrasónicas no son las más adecuadas [15]

Se contrastó la normalidad de los datos a través del método Shapiro-Wilk, se descartaron datos por el criterio de Chauvenet y se aplicó análisis de varianza ANOVA. La correlación de Pearson hallada fue lineal a excepción de la combinación 5 [16].

\section{III.RESULTADOS}

a) Los resultados de granulometrías típicas de los materiales utilizados se presentan a continuación:

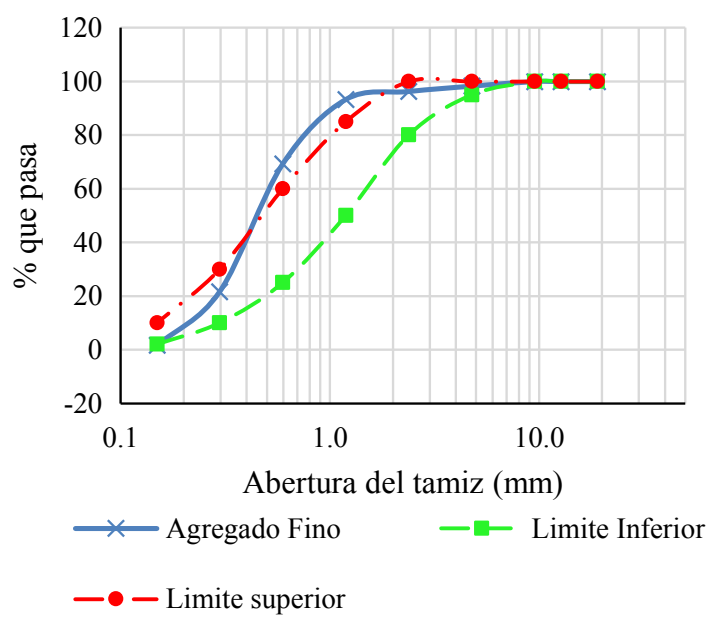

Fig. 1. Arena fina del Río Sinú Fuente: Autor

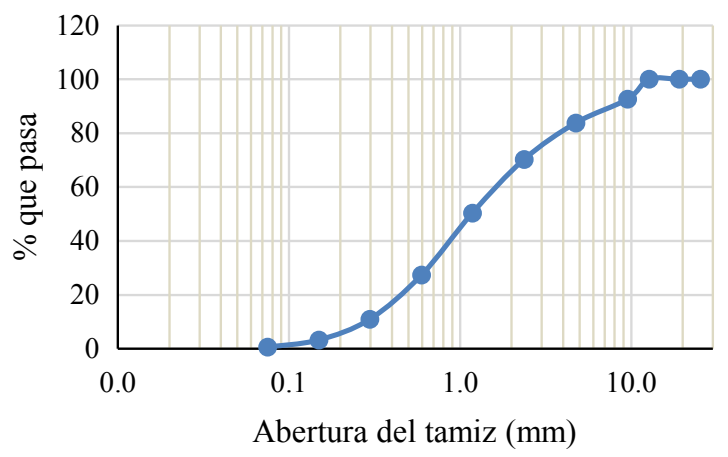

Fig. 2. Arena gruesa del Río Sinú Fuente: Autor

De las figuras 1 y 2 se obtuvo el módulo de finura para las arenas finas y gruesas los cuales fueron 2.19 y 3.61, respectivamente, valores típicos para estas clases de arena.
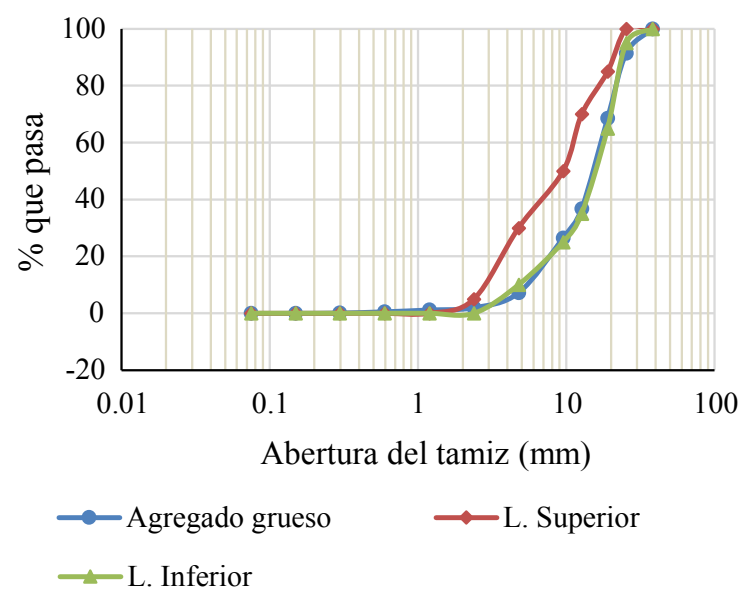

Fig. 3. Canto rodado (grava) del Río Sinú Fuente: Autor

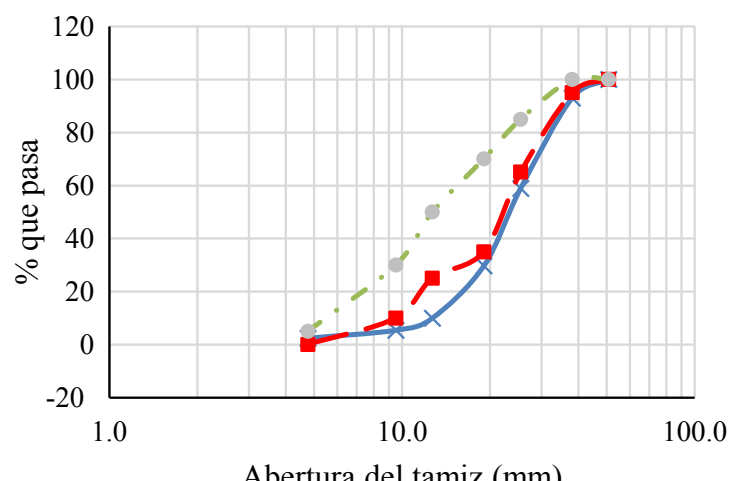

$$
\begin{aligned}
& * \text { Cantera Siberia } \rightarrow-\text { L. Inferior } \\
& -0-\text { L. Superior }
\end{aligned}
$$

Fig. 4. Triturado (Caliza) cantera La Siberia Fuente: Autor

Tabla II. Velocidades de pulso obtenidas

\begin{tabular}{|c|c|c|}
\hline Grupo & $\begin{array}{c}\text { Combinación de } \\
\text { agregados }\end{array}$ & $\begin{array}{c}\text { Rango de lecturas } \\
\text { para resistencias } \\
\text { de 28 MPa (m/s) }\end{array}$ \\
\hline 1 & $\begin{array}{c}\text { Arena fina del río Sinú con } \\
\text { caliza de cantera Siberia }\end{array}$ & $4110-4202$ \\
\hline 2 & $\begin{array}{c}\text { Arena fina con Canto } \\
\text { rodado (río Sinú) }\end{array}$ & $3830-4075$ \\
\hline 3 & $\begin{array}{c}\text { Arena fina con Canto } \\
\text { rodado (río Sinú) y caliza de } \\
\text { la cantera Siberia }\end{array}$ & $3570-4470$ \\
\hline 4 & $\begin{array}{c}\text { Arena gruesa del río Sinú } \\
\text { con caliza de la cantera } \\
\text { Siberia }\end{array}$ & $3930-4010$ \\
\hline 5 & $\begin{array}{c}\text { Arena gruesa con canto } \\
\text { rodado (río Sinú) }\end{array}$ & $3630-3760$ \\
\hline 6 & $\begin{array}{c}\text { Arena gruesa con Canto } \\
\text { rodado (río Sinú) y caliza de } \\
\text { la cantera Siberia }\end{array}$ & $4010-4800$ \\
\hline
\end{tabular}

Fuente: Autor 
Las figuras 3 y 4 muestran la granulometría de los agregados gruesos (canto rodado y caliza), lo cuales cumplen con los límites superiores e inferiores indicados en la Norma NTC - 174 [19].

b) En el cuadro 2 se resumen las velocidades de onda obtenidas para cada una de las diferentes dosificaciones.

c) Las gráficas de correlación para las diferentes combinaciones de materiales pétreos y resistencia se relacionan a continuación:

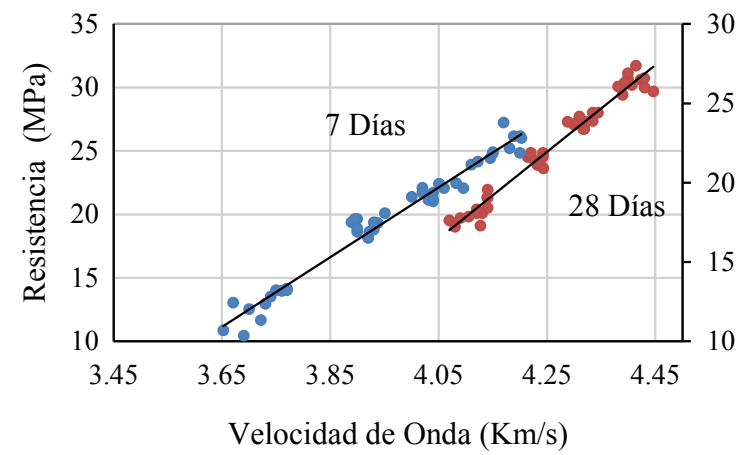

Fig. 5. Resistencia a la compresión a 7 y 28 días combinación 1: arena fina y caliza Fuente: Autor

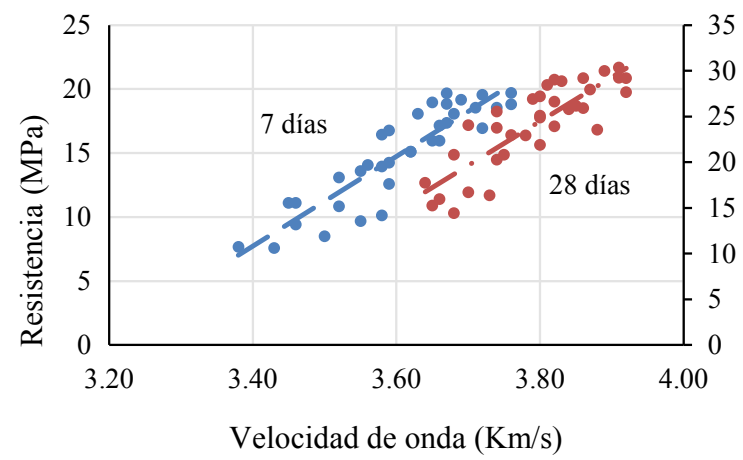

Fig. 6. Resistencia a la compresión a 7 y 28 días combinación 2: arena fina y Canto rodado río Sinú Fuente: Autor

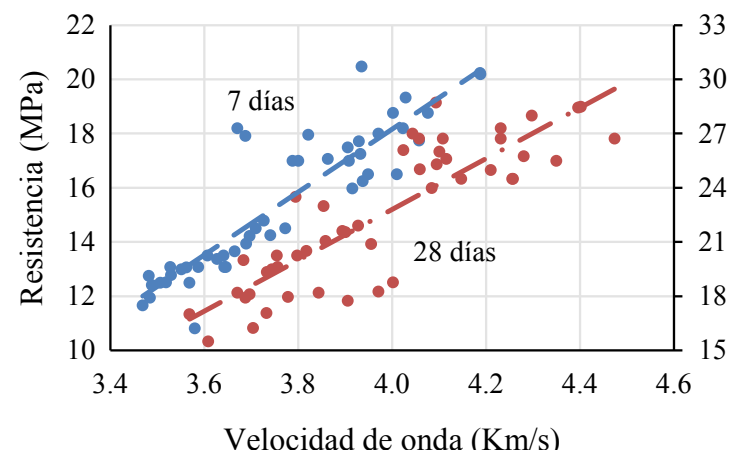

Fig. 7. Resistencia a la compresión a 7 y 28 días combinación 3: arena fina y caliza más canto rodado río Sinú Fuente: Autor

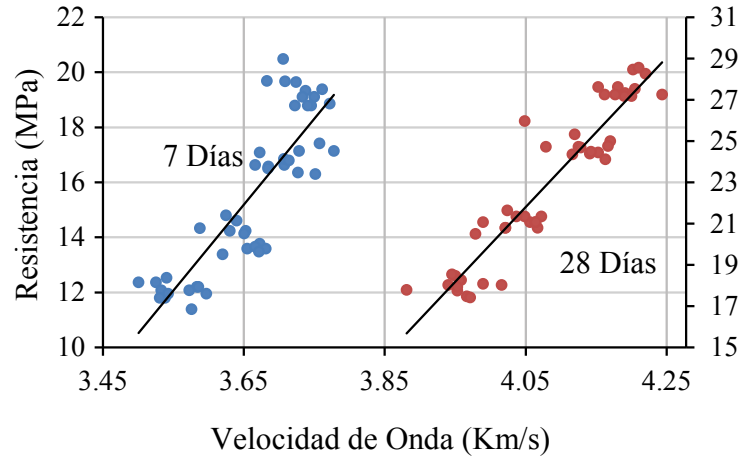

Fig. 8. Resistencia a la compresión a 7 y 28 días combinación 4: arena gruesa y caliza Fuente: Autor

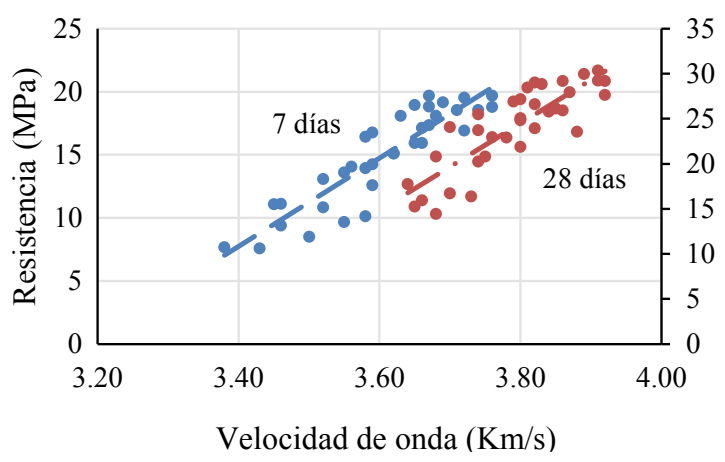

Fig. 9. Resistencia a la compresión a 7 y 28 días combinación 5: arena gruesa y Canto rodado río Sinú Fuente: Autor

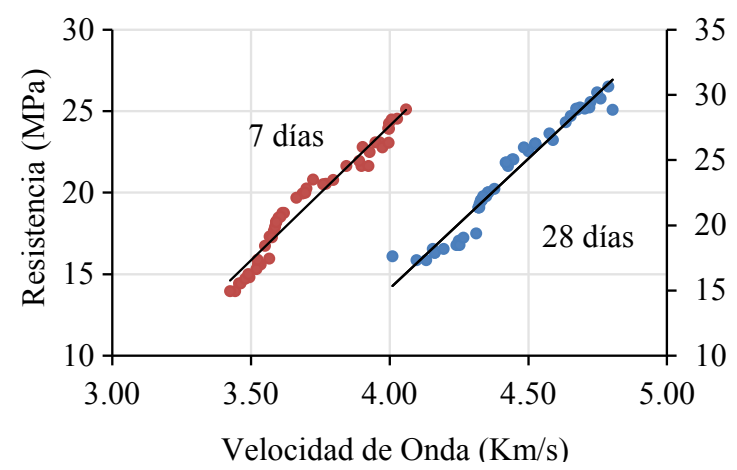

Fig. 10. Resistencia a la compresión a 7 y 28 días combinación 6: arena gruesa y caliza más Canto rodado río Sinú

Fuente: Autor

Las ecuaciones de correlación resultantes en el presente trabajo con sus respectivos factores de correlación se obtuvieron de las figuras 5, 6, $7,8,9$ y 10 , y se muestran a continuación en los cuadros III y IV, para 7 y 28 días respectivamente: 
TABla III. ECUACIONES dE CORRELACIÓN PARA 7 díAS

\begin{tabular}{|cc|c|}
\hline Grupo & Ecuación & $\mathbf{R}^{2}$ \\
\hline 1 & $\mathrm{y}=21.9565 \mathrm{x}-69.216$ & 0.9700 \\
\hline 2 & $\mathrm{y}=17.80 \mathrm{x}-53.90$ & 0.7920 \\
\hline 3 & $\mathrm{y}=11.612 \mathrm{x}-28.284$ & 0.8012 \\
\hline 4 & $\mathrm{y}=31.219 \mathrm{x}-98.762$ & 0.7325 \\
\hline 5 & $\mathrm{y}=35.037 \mathrm{x}-111.39$ & 0.8156 \\
\hline 6 & $\mathrm{y}=15.609 \mathrm{x}-41.935$ & 0.9526 \\
\hline
\end{tabular}

Fuente: Autor

TABla IV. Ecuaciones de CORRELación PARA 28 días

\begin{tabular}{|cc|c|}
\hline Grupo & Ecuación & $\mathbf{R}^{2}$ \\
\hline 1 & $\mathrm{y}=34.219 \mathrm{x}-120.5$ & 0.9685 \\
\hline 2 & $\mathrm{y}=40.72 \mathrm{x}-141.2$ & 0.8960 \\
\hline 3 & $\mathrm{y}=14.068 \mathrm{x}-33.466$ & 0.7374 \\
\hline 4 & $\mathrm{y}=36.245 \mathrm{x}-124.99$ & 0.8844 \\
\hline 5 & $\mathrm{y}=48.269 \mathrm{x}-158.94$ & 0.7154 \\
\hline 6 & $\mathrm{y}=19.948 \mathrm{x}-64.654$ & 0.9565 \\
\hline
\end{tabular}

Fuente: Autor

\section{Conclusiones}

Teniendo en cuenta los resultados obtenidos de acuerdo a las diferentes combinaciones presentadas, se deduce que conociendo las proporciones y procedencia de los materiales empleados en la mezcla para la elaboración del concreto, se puede realizar una estimación de la resistencia de dicho concreto utilizando un equipo de ultrasonido, sin realizar ensayos destructivos.

Sin embargo, no siempre se cuenta con los datos relativos a la procedencia de los materiales, lo cual impediría aplicar esta metodología para encontrar la resistencia de los especímenes.

Una vez hallada dicha correlación entre los dos métodos, se podrán hacer ensayos ultrasónicos a estructuras que se quieran potencializar a través de estudios de vulnerabilidad sísmica [6], construidas con materiales similares a los de este estudio y de esta manera predecir con certeza la resistencia del concreto sin necesidad de destruir los elementos.

\section{REFERENCIAS}

[1] A. O. De La Haza, A. A. Samokrutov, and P. A. Samokrutov, "Assessment of concrete structures using the Mira and Eyecon ultrasonic shear wave devices and the SAFTC image reconstruction technique," Constr. Build. Mater., vol. 38, pp. 1276-1291, 2013.
[2] M. A. Kewalramani and R. Gupta, "Concrete compressive strength prediction using ultrasonic pulse velocity through artificial neural networks," Autom. Constr., vol. 15, no. 3, pp. 374-379, 2006.

[3] P. Antonaci, C. L. E. Bruno, A. S. Gliozzi, and M. Scalerandi, "Monitoring evolution of compressive damage in concrete with linear and nonlinear ultrasonic methods," Cem. Concr. Res., vol. 40, no. 7, pp. 1106$1113,2010$.

[4] K. Komloŝ, S. Popovics, T. Nürnbergerová, B. Babál, and J. S. Popovics, "Ultrasonic pulse velocity test of concrete properties as specified in various standards," Cem. Concr. Compos., vol. 18, no. 5, pp. 357-364, Jan. 1996.

[5] J. Berriman, P. Purnell, H. D.A. y A. Neild, "Humidity and Aggregate content correction factors for air-coupled ultrasonic evaluation of concrete," Ultrasonics, no. 43, pp. 211-217, 2005.

[6] Asociación Colombiana de Ingeniería Sísmica, Reglamento Colombiano de Construcciones Sismo Resistentes, Bogota: AIS, 2010.

[7] P. J. J. T. y J. Kim, "Detection of delaminationin concrete using ultrasonic pulse velocity test," Constr. Build. Mater. no 26, pp. 574-582, 2012.

[8] INVIAS, «INVIAS,» 0305 2013. [En línea]. Available: http://www.invias.gov.co/index.php/documentos-tecnicos-izq/cat_view/11-documentos-tecnicos/68-especificaciones-generales-de-construccion-y-normas-de-ensayo-para-materiales-de-carreteras. [Último acceso: 20 05 2013].

[9] Instituto Colombiano de Normas Técnicas y Certificación, ICONTEC, Norma Técnica Colombiana NTC 237 - Ingeniería Civil y Arquitectura. Método para determinar la densidad y la absorción del agregado fino, Bogotá: ICONTEC, 1995.

[10] Instituto Colombiano de Normas Técnicas y Certificación, ICONTEC, Norma Técnica Colombiana NTC 176 - Ingeniería Civil y Arquitectura. Método de ensayo para determinar la densidad y la absorción del agregado grueso, Bogotá: ICONTEC, 1995.

[11] E. Ohdaira y N. Masuzawa, "Water content and its effect on ultrasound propagation in concrete - the possibility of NDE," Ultrasonics, no. 38, pp. 546-552, 2000.

[12] R. G. Solís Carcaño, E. I. Moreno y W. R. Castillo Pak, "Predicción de la resistencia del concreto con base en la velocidad de pulso ultrasónico y un índice de calidad de los agregados" Ingeniería Revista Académica, vol. 8, $\mathrm{n}^{\circ}$ 002, pp. 41-52, 2004.

[13] H. Y. Qasrawi and I. A. Marie, "The use of USPV to anticipate failure in concrete under compression," Cem. Concr. Res., vol. 33, no. 12, pp. 2017-2021, 2003.

[14] M. T. Liang and J. Wu, "Theoretical elucidation on the empirical formulae for the ultrasonic testing method for concrete structures," Cem. Concr. Res., vol. 32, no. 11, pp. 1763-1769.

[15] H. K. Lee, K. M. Lee, Y. H. Kim, H. Yim, and D. B. Bae, "Ultrasonic in-situ monitoring of setting process of high-performance concrete," Cem. Concr. Res., vol. 34, no. 4, pp. 631-640, 2004.

[16] L. J. Devore, Probabilidad y estadística para ingeniería y ciencias, Thompson, 2005

[17] R. Solís y E. I. Moreno, "Evaluation of concretemade with crushed limestone aggregate based on ultrasonic pulse velocity," Construction and Building Materials, no. 22 , pp. $1225-1231,2008$ 